\title{
Statistical Analyses of the Forms of the Glaciers in the Khumbu Himal*
}

\author{
Yoshiyuki Fujii** and Keiji Higuchi***
}

\begin{abstract}
Statistical analyses were performed on the relation between the glacier forms and the topographic conditions of the glacier basins, using an inventory of the glaciers in the Khumbu Himal where climatic conditions can be considered as nearly uniform in the Great Himalayas.

An apparent difference was found between the dimensions of glaciers without debris (C-type glaciers) and those covered with debris (D-type glaciers), the latter being 15 times larger in area and 5 times longer in length on the average.

The correlation between the dimensions of glaciers and their basins is better in the case of glaciers covered with ablation moraine than glaciers without ablation moraine. The large-sized glaciers, having large area, long length, and high upper limit, develop in bigger basins with higher summits, longer basin ridges and wider valleys. However the positions of the lower limits of the glaciers are independent of the dimensions of the glacier basin.

There are good correlations between glacier area and basin area, and between debris covered area and rock face area. Therefore, it would be reasonable to consider that the total precipitation in the area of basin above the firn line controls the size of the glacier, and the debris on the glacier surface were supplied from the rock face of the basin.

After multiple regression analysis by forward selection, elements representing the form of the glaciers were related to elements representing the form of the basins by a regression equation.
\end{abstract}

\section{Introduction}

Various types and forms of the glaciers in the Himalayas indicate that multiple conditions of both climates and landforms affect on glacier formation. But, since the climatic conditions can be considered as nearly uniform in a small area, the topographical conditions of glacier basins are predominant factors to decide glacier forms and types in that area.

The Khumbu region in eastern Nepal Himalayas was chosen for a study on the relationship between the forms of the glaciers and those of the glacier basins for several reasons: (a) this test area covers the highest mountain group, including Mt. Sagarmatha (Everest, 8848m), Mt. Lotse

* Glaciological Expedition of Nepal, Contribution No. 31

** Water Research Institute, Nagoya University (present address, National Institute of Polar Research, Kaga 1-9, Itabashi-ku, Tokyo 173)

*** Water Research Institute, Nagoya University, Furo-cho, Chikusa-ku, Nagoya 464 (8510m) and Mt. Cho Oyu (8153m) and is one of the most glacierized areas in the Nepal Himalayas; (b) various field studies were carried out on the glaciers and climates and a large number of photographs were taken during the expedition; (c) maps of different scale are available; (d) a pilot study for an inventory of the glaciers was reported by Müller (1970).

This paper discusses preliminary results of the statistical analyses of the forms of the glaciers in connection with the forms of glacier basins.

\section{Inventory of the glaciers and their basins}

As a part of the IHD 1965-1974, the International Commission of Snow and Ice (ICSI) of the International Association of Scientific Hydrology (IASH) prepared the report "Perennial ice and snow masses; a guide for compilation and assemblage of data for a world inventory" (Unesco/Iash, 1970), in which an inventory of glaciers in the Mount Sagarmatha (Everest) region was reported by Müller (1970) as one of the pilot studies.

An inventory of glaciers in a small area where 


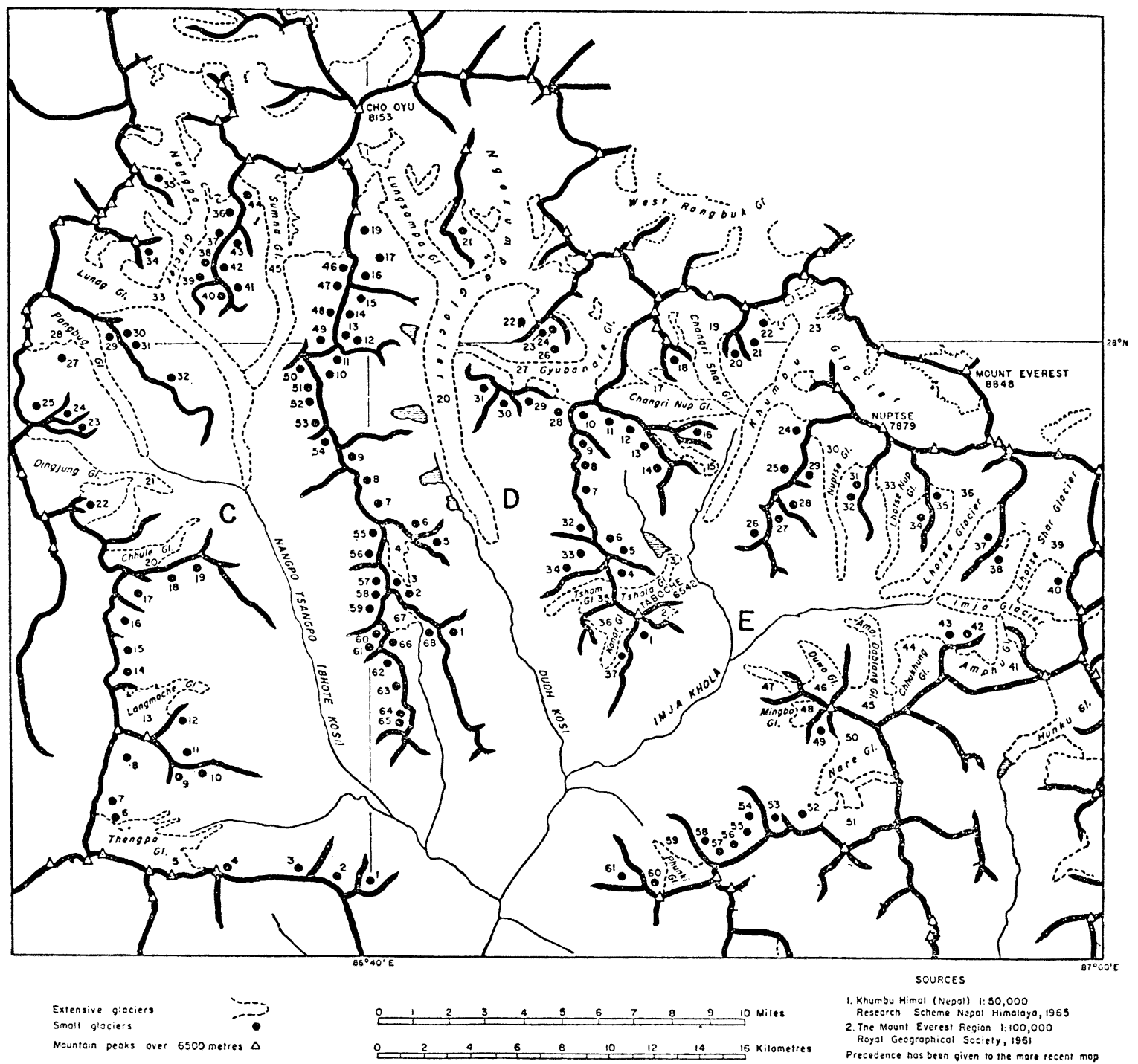

Fig. 1. Index map of the glacier inventory of Khumbu Himal. Quoted From Müller (1970).

climatic conditions are nearly uniform can be used for a study of the relation between glacier form and the topographical conditions of the basins in which the glaciers lie.

For such a study, ten elements which seem to represent glacier form and eight elements to represent glacier basins were selected as follows.

a) Elements of glacier form
G 1 Upper limit (m)
G 2 Lower limit (m)
G 3 'Exposed' limit
G 4 Debris covered area $\left(\mathrm{km}^{2}\right)$
G 5 Total area $\left(\mathrm{km}^{2}\right)$
G 6 Length (m)

G 7 Debris covered area ratio (G4/G5) $(\%)$

G 8 Altitudinal range $(\mathrm{G} 1-\mathrm{G} 2)$, (m)

G 9 Gradient (G8/G6)

G10 Square root of total area (m)

b) Elements of glacier basin

T 1 Maximum height (m)

T 2 Length of ridge above $5500 \mathrm{~m}(\mathrm{~m})$

T 3 Width of basin at $5500 \mathrm{~m}(\mathrm{~m})$

T 4 Area of basin above $5500 \mathrm{~m}\left(\mathrm{~km}^{2}\right)$

T 5 Area of rock face above $5500 \mathrm{~m}\left(\mathrm{~km}^{2}\right)$

T 6 Basin shape index (T2/T3)

T 7 Rock face area ratio (T5/T4), (\%)

Measurments of the above mentioned elements of glaciers and their basins were carried out 


\begin{tabular}{|c|c|c|c|c|c|c|c|c|c|c|}
\hline & $\leftrightarrow 1$ & 62 & $G 2$ & 64 & 65 & 60 & 67 & $\begin{array}{ll}68 \\
\text { D }\end{array}$ & $G O$ & G10 \\
\hline $\begin{array}{ll}C & 1 \\
C & 2 \\
C & 3 \\
C & 4 \\
C & b \\
C & b\end{array}$ & $\begin{array}{l}4940 \\
5240 \\
51100 \\
5900 \\
5700 \\
5200\end{array}$ & $\begin{array}{l}4640 \\
4700 \\
4620 \\
5401 \\
4300 \\
4879\end{array}$ & $\begin{array}{l}4540 \\
4700 \\
4620 \\
5460 \\
4470 \\
4900\end{array}$ & $\begin{array}{l}n .00 \\
0.00 \\
9.00 \\
0.00 \\
1.16 \\
0.00\end{array}$ & $\begin{array}{l}0.16 \\
0.20 \\
0.25 \\
0.23 \\
5.31 \\
0.15\end{array}$ & $\begin{array}{r}200 \\
500 \\
400 \\
400 \\
4000 \\
900\end{array}$ & $\begin{array}{r}0 \\
0 \\
0 \\
0 \\
21 \\
0\end{array}$ & $\begin{array}{r}300 \\
540 \\
480 \\
500 \\
1560 \\
300\end{array}$ & $\begin{array}{l}1.5 n \\
1.00 \\
0.80 \\
1.25 \\
0.41 \\
0.32\end{array}$ & $\begin{array}{r}400 \\
529 \\
500 \\
479 \\
2304 \\
387\end{array}$ \\
\hline $\begin{array}{lr}C & 0 \\
C & y \\
C & 10 \\
C & 11 \\
C & 11 \\
C & 12 \\
C & 13 \\
C & 13 \\
C & 15 \\
C & 16 \\
C & 17 \\
C & 17 \\
C & 18 \\
C & 19\end{array}$ & $\begin{array}{l}6100 \\
6100 \\
5360 \\
5440 \\
5860 \\
5600 \\
5750 \\
5840 \\
5690 \\
5800 \\
5860 \\
5600\end{array}$ & $\begin{array}{l}5100 \\
5119 \\
5040 \\
4899 \\
5400 \\
4410 \\
4960 \\
5100 \\
5100 \\
5250 \\
5100 \\
5280\end{array}$ & $\begin{array}{l}5160 \\
5119 \\
5040 \\
4899 \\
5400 \\
4400 \\
4900 \\
5100 \\
5100 \\
5259 \\
5160 \\
5280\end{array}$ & $\begin{array}{l}0.16 \\
0.00 \\
0.00 \\
0.00 \\
0.00 \\
0.52 \\
0.00 \\
0.00 \\
0.20 \\
0.00 \\
0.00 \\
0.00\end{array}$ & $\begin{array}{l}2.04 \\
0.48 \\
0.24 \\
1.06 \\
0.04 \\
2.09 \\
0.39 \\
0.80 \\
1.00 \\
1.51 \\
1.37 \\
0.82\end{array}$ & $\begin{array}{r}1400 \\
900 \\
600 \\
1200 \\
200 \\
3300 \\
1100 \\
1100 \\
1400 \\
2000 \\
3000 \\
1500\end{array}$ & $\begin{array}{r}7 \\
0 \\
0 \\
0 \\
0 \\
24 \\
0 \\
0 \\
0 \\
0 \\
0 \\
0\end{array}$ & $\begin{array}{r}940 \\
979 \\
320 \\
580 \\
400 \\
1190 \\
800 \\
740 \\
580 \\
540 \\
700 \\
320\end{array}$ & $\begin{array}{l}0.52 \\
1.08 \\
0.53 \\
0.48 \\
2.30 \\
0.36 \\
0.72 \\
0.67 \\
0.41 \\
0.27 \\
0.23 \\
0.21\end{array}$ & $\begin{array}{r}1428 \\
699 \\
489 \\
1029 \\
200 \\
1445 \\
616 \\
894 \\
1000 \\
1228 \\
1170 \\
905\end{array}$ \\
\hline $\begin{array}{ll}C & 21 \\
C & 22 \\
C & 23 \\
C & 24 \\
C & 25\end{array}$ & $\begin{array}{l}5850 \\
2200 \\
5630 \\
56110 \\
5790\end{array}$ & $\begin{array}{l}4850 \\
5300 \\
5410 \\
5390 \\
5160\end{array}$ & $\begin{array}{l}5130 \\
5300 \\
5410 \\
5390 \\
5240\end{array}$ & $\begin{array}{l}2.35 \\
0.00 \\
0.00 \\
0.00 \\
0.70\end{array}$ & $\begin{array}{l}6.97 \\
0.71 \\
0.38 \\
0.18 \\
2.61\end{array}$ & $\begin{array}{r}7300 \\
1400 \\
1400 \\
500 \\
4500\end{array}$ & $\begin{array}{r}33 \\
0 \\
0 \\
0 \\
20\end{array}$ & $\begin{array}{r}1000 \\
900 \\
270 \\
210 \\
620\end{array}$ & $\begin{array}{l}0.13 \\
0.64 \\
0.19 \\
0.42 \\
0.13\end{array}$ & $\begin{array}{r}2640 \\
847 \\
616 \\
424 \\
1615\end{array}$ \\
\hline $\begin{array}{ll}C & 27 \\
C & 28 \\
C & 29 \\
C & 30 \\
C & 31 \\
C & 32 \\
C & 33 \\
C & 34 \\
C & 35 \\
C & 30 \\
C & 37 \\
C & 38 \\
C & 39 \\
C & 40 \\
C & 41 \\
C & 42 \\
C & 43 \\
C & 44 \\
C & 45 \\
C & 46 \\
C & 47 \\
C & 48 \\
C & 49 \\
C & 50 \\
C & 51 \\
C & 52 \\
C & 53 \\
C & 54 \\
C & 55 \\
C & 56 \\
C & 57 \\
C & 58 \\
C & 59 \\
C & 60 \\
C & 61 \\
C & 62 \\
C & 63 \\
C & 64 \\
C & 65 \\
C & 65 \\
C & 60 \\
C & 67 \\
C & 58\end{array}$ & $\begin{array}{l}5760 \\
6600 \\
57130 \\
5600 \\
5600 \\
5570 \\
6900 \\
6000 \\
5840 \\
5680 \\
6200 \\
5630 \\
5390 \\
5460 \\
5460 \\
5650 \\
5610 \\
5840 \\
7200 \\
5580 \\
5380 \\
5650 \\
5950 \\
5880 \\
5640 \\
5640 \\
5480 \\
5855 \\
5650 \\
5800 \\
5880 \\
5700 \\
5500 \\
5600 \\
5380 \\
5700 \\
5560 \\
5520 \\
5540 \\
5800 \\
5840 \\
5440\end{array}$ & $\begin{array}{l}5369 \\
5020 \\
5340 \\
5270 \\
5420 \\
5300 \\
4500 \\
5440 \\
5310 \\
5259 \\
5350 \\
5310 \\
5220 \\
5310 \\
5330 \\
5450 \\
5380 \\
5481 \\
4840 \\
5230 \\
5119 \\
5390 \\
5240 \\
5600 \\
5320 \\
5240 \\
5190 \\
5440 \\
5240 \\
5340 \\
5020 \\
5360 \\
5100 \\
5210 \\
5200 \\
5350 \\
5340 \\
5100 \\
5270 \\
5380 \\
5350 \\
5280\end{array}$ & $\begin{array}{l}5390 \\
5250 \\
5340 \\
5270 \\
5420 \\
5300 \\
5119 \\
5440 \\
5310 \\
5250 \\
5350 \\
5310 \\
5220 \\
5310 \\
5330 \\
5450 \\
5380 \\
5480 \\
5210 \\
5230 \\
5119 \\
5340 \\
5240 \\
5000 \\
5320 \\
5240 \\
5190 \\
5440 \\
5240 \\
5340 \\
5020 \\
5350 \\
5100 \\
5210 \\
5200 \\
5350 \\
5340 \\
5100 \\
5270 \\
5380 \\
5350 \\
5280\end{array}$ & $\begin{array}{r}0.12 \\
3.45 \\
0.00 \\
0.00 \\
0.00 \\
0.00 \\
13.40 \\
0.00 \\
0.00 \\
0.00 \\
0.00 \\
0.00 \\
0.00 \\
0.00 \\
0.00 \\
0.00 \\
0.00 \\
0.00 \\
5.06 \\
0.00 \\
0.00 \\
0.00 \\
0.00 \\
0.00 \\
0.00 \\
0.00 \\
0.00 \\
0.00 \\
0.00 \\
0.00 \\
0.00 \\
0.00 \\
0.00 \\
0.00 \\
0.00 \\
0.00 \\
0.00 \\
0.00 \\
0.00 \\
0.00 \\
0.00 \\
0.00\end{array}$ & $\begin{array}{r}0.62 \\
5.65 \\
0.19 \\
0.20 \\
0.09 \\
0.44 \\
30.30 \\
0.24 \\
0.50 \\
0.51 \\
0.24 \\
0.04 \\
0.14 \\
0.07 \\
0.07 \\
0.10 \\
0.15 \\
0.71 \\
14.54 \\
0.32 \\
0.29 \\
0.06 \\
0.55 \\
0.009 \\
0.31 \\
0.15 \\
0.15 \\
0.17 \\
0.14 \\
0.22 \\
0.47 \\
0.07 \\
0.09 \\
0.17 \\
0.06 \\
0.17 \\
0.06 \\
0.18 \\
0.13 \\
0.35 \\
1.29 \\
0.04\end{array}$ & $\begin{array}{r}1400 \\
8500 \\
1000 \\
100 \\
400 \\
000 \\
19200 \\
100 \\
1300 \\
1400 \\
1000 \\
500 \\
700 \\
500 \\
500 \\
100 \\
400 \\
1000 \\
9500 \\
1300 \\
500 \\
300 \\
1400 \\
400 \\
100 \\
600 \\
500 \\
800 \\
800 \\
1000 \\
1300 \\
400 \\
400 \\
1000 \\
300 \\
500 \\
300 \\
500 \\
900 \\
700 \\
1200 \\
300\end{array}$ & $\begin{array}{r}19 \\
61 \\
0 \\
0 \\
0 \\
0 \\
44 \\
0 \\
0 \\
0 \\
0 \\
0 \\
0 \\
0 \\
0 \\
0 \\
0 \\
0 \\
38 \\
0 \\
0 \\
0 \\
0 \\
0 \\
0 \\
0 \\
0 \\
0 \\
0 \\
0 \\
0 \\
0 \\
0 \\
0 \\
0 \\
0 \\
0 \\
0 \\
0 \\
0 \\
0 \\
0\end{array}$ & $\begin{array}{r}390 \\
1580 \\
440 \\
330 \\
180 \\
220 \\
2400 \\
560 \\
510 \\
420 \\
450 \\
320 \\
170 \\
150 \\
130 \\
200 \\
230 \\
360 \\
2360 \\
350 \\
260 \\
260 \\
710 \\
280 \\
320 \\
400 \\
290 \\
415 \\
410 \\
460 \\
460 \\
340 \\
260 \\
390 \\
180 \\
350 \\
220 \\
420 \\
270 \\
420 \\
530 \\
160\end{array}$ & $\begin{array}{l}0.27 \\
0.14 \\
0.44 \\
0.55 \\
0.45 \\
0.24 \\
0.17 \\
0.93 \\
0.43 \\
0.30 \\
0.85 \\
0.64 \\
0.24 \\
0.30 \\
0.26 \\
0.33 \\
0.57 \\
0.22 \\
0.24 \\
0.26 \\
0.43 \\
0.86 \\
0.50 \\
0.70 \\
0.53 \\
0.66 \\
0.58 \\
0.69 \\
0.51 \\
0.46 \\
0.66 \\
0.85 \\
0.65 \\
0.65 \\
0.90 \\
0.70 \\
0.73 \\
0.70 \\
0.30 \\
0.60 \\
0.29 \\
0.53\end{array}$ & $\begin{array}{r}787 \\
2376 \\
435 \\
447 \\
299 \\
699 \\
5504 \\
489 \\
707 \\
714 \\
509 \\
282 \\
374 \\
264 \\
764 \\
316 \\
387 \\
442 \\
3813 \\
565 \\
538 \\
244 \\
741 \\
299 \\
556 \\
387 \\
346 \\
412 \\
400 \\
469 \\
685 \\
264 \\
299 \\
212 \\
412 \\
244 \\
412 \\
244 \\
424 \\
300 \\
599 \\
1135 \\
200\end{array}$ \\
\hline
\end{tabular}

\begin{tabular}{|c|c|c|c|c|c|c|c|c|c|c|}
\hline D 1 & 5540 & 5080 & 5020 & 1.00 & 0.11 & 500 & 0 & 500 & $1.0 n$ & 331 \\
\hline D 2 & 5620 & 5100 & 5240 & $9 . \cap 3$ & $0.1 \mathrm{n}$ & roo & 18 & 520 & 0.65 & 400 \\
\hline & 5720 & $5 ? 80$ & 5280 & 0.00 & 0.05 & 500 & 0 & 440 & 0.89 & 223 \\
\hline D 4 & 5900 & 5140 & 5180 & 0.24 & $1.3 \mathrm{H}$ & 2700 & 20 & H2O & 0.30 & 1174 \\
\hline D & 5470 & 5000 & 5119 & 0.03 & 0.09 & 500 & 33 & 340 & 0.68 & 290 \\
\hline 6 & 5400 & 4940 & 4940 & 11.06 & 0.15 & Soo & 40 & 460 & 0.76 & 387 \\
\hline 7 & 5680 & 5140 & 5140 & 0.01 & 1.03 & 1500 & 6 & 540 & 0.36 & 1014 \\
\hline D 8 & $55>0$ & 5300 & 5300 & 0.00 & 0.06 & 400 & 0 & 220 & 0.55 & 244 \\
\hline & 5620 & 5180 & 5180 & 0.03 & $0.4 \mathrm{~h}$ & 1500 & 0 & 440 & 0.29 & 678 \\
\hline D 10 & 5640 & 5300 & 5300 & 0.00 & 0.90 & 1500 & 0 & 340 & 0.22 & 948 \\
\hline D 11 & 5640 & 5340 & 5340 & $n \cdot n_{1}$ & 0.59 & 1300 & 0 & 340 & 0.26 & 768 \\
\hline D 12 & 5720 & 5420 & 5420 & 0.00 & 0.24 & 1000 & 0 & 300 & 0.30 & 489 \\
\hline 0 is & 5520 & 5300 & 5300 & 0.00 & 0.18 & 300 & 0 & 220 & 0.73 & 316 \\
\hline D 14 & $58>0$ & 5540 & 5540 & $0.0 n$ & $0.0 \mathrm{n}$ & 800 & 0 & 280 & 0.46 & 244 \\
\hline$D$ is & 5040 & $536 n$ & 5360 & 0.00 & 0.11 & non & 0 & 320 & 0.53 & 331 \\
\hline 016 & 5500 & 5310 & 5310 & 11.00 & 0.94 & 1000 & 0 & 250 & 0.13 & 969 \\
\hline D 17 & $53>0$ & 5350 & 5350 & n.no & 0.08 & 400 & 0 & 170 & 0.47 & 282 \\
\hline$D 19$ & 6100 & $532 n$ & 5320 & 0.00 & 0.85 & 1400 & 0 & 780 & 0.55 & 927 \\
\hline & 7200 & 4690 & 5009 & 20.57 & 03.33 & 21000 & 32 & 2510 & 0.11 & 7958 \\
\hline D ? 1 & 6059 & 5330 & 5330 & 0.00 & 0.57 & IRON & $\begin{array}{r}32 \\
0\end{array}$ & 730 & 0.40 & 754 \\
\hline D 22 & 5600 & 5240 & 5240 & 0.01 & 0.59 & 1000 & 0 & 360 & 0.36 & 768 \\
\hline D 23 & 5600 & 5590 & 5500 & 0.00 & 0.15 & 500 & 0 & 210 & $0.4 ?$ & 387 \\
\hline D 24 & 5610 & 5480 & 5480 & 0.00 & 0.10 & 700 & 0 & 120 & 0.17 & 316 \\
\hline D 20 & 5470 & $538 n$ & 5380 & 0.00 & 0.15 & 400 & 0 & 90 & 0.11 & 387 \\
\hline & 6300 & 5000 & 5259 & 4.51 & 13.17 & 10500 & 34 & 1300 & 0.12 & 3529 \\
\hline $\begin{array}{l}\text { D } 28 \\
\text { D. }\end{array}$ & 5520 & 5180 & 5180 & 0.00 & 0.64 & 1700 & 0 & 340 & 0.20 & 800 \\
\hline D 29 & 5800 & 5390 & 5390 & $0 . n 0$ & 0.35 & 1000 & 0 & 410 & 0.41 & 591 \\
\hline 030 & 6000 & 5480 & 5440 & 0.0 .1 & 0.15 & 500 & 0 & 520 & 1.04 & 387 \\
\hline 31 & 5540 & 3270 & 5270 & n.nu & 0.07 & 500 & 0 & 270 & 0.54 & 264 \\
\hline 032 & 5480 & 5000 & 5040 & 0.06 & 0.37 & 900 & 16 & 480 & 0.53 & 608 \\
\hline D 33 & 5600 & 5020 & 5020 & 0.00 & $0.5 \mathrm{~A}$ & 1000 & 0 & 580 & 0.58 & 748 \\
\hline D 34 & 5450 & 5180 & 5180 & $0_{0} .00$ & 0.04 & 300 & 0 & 270 & 0.90 & 200 \\
\hline D 35 & 5500 & 4880 & 5119 & 1.04 & 1,72 & 3100 & no & 620 & 0.20 & 1311 \\
\hline $\begin{array}{lll}0 & 30\end{array}$ & 5600 & 4800 & 5100 & 1.13 & 1.37 & 2200 & $\begin{array}{l}n 0 \\
82\end{array}$ & $\begin{array}{l}0<0 \\
800\end{array}$ & 0.28 & 1170 \\
\hline D 37 & 5320 & 5090 & 5090 & 0.00 & 0.14 & 900 & 0 & 230 & 0.25 & 374 \\
\hline
\end{tabular}

Fig. 2. Inventory of the glaciers in the Khumbu region, Bhote Kosi and Dudh Kosi. 


\begin{tabular}{|c|c|c|c|}
\hline 1 & 8542 & $200 \mathrm{C}$ & 1100 \\
\hline 2 & 6542 & 2000 & 900 \\
\hline 3 & $\begin{array}{l}6542 \\
6440\end{array}$ & $\begin{array}{l}2500 \\
3000\end{array}$ & 1300 \\
\hline 5 & 6440 & 1500 & 1100 \\
\hline 6 & 6440 & 2300 & 1100 \\
\hline 7 & 5939 & 1800 & 1000 \\
\hline$B$ & 5729 & 1000 & 700 \\
\hline $\begin{array}{ll}E & 0 \\
E & 0\end{array}$ & 5065 & 1600 & 800 \\
\hline E 10 & 6164 & 4000 & 700 \\
\hline E 11 & 6169 & 2000 & 800 \\
\hline E 12 & 6145 & 1900 & 700 \\
\hline$E 13$ & 6145 & 2600 & 1400 \\
\hline$E 14$ & 6119 & 2100 & 900 \\
\hline E 15 & 6145 & 4800 & 1000 \\
\hline $\begin{array}{lll}C & 16\end{array}$ & 5940 & 1800 & on 0 \\
\hline E 17 & 6853 & 13000 & 2000 \\
\hline$E$ L & 6549 & 3000 & 1000 \\
\hline E 19 & 7145 & 9300 & 1600 \\
\hline$E 20$ & 6119 & $210 n$ & 900 \\
\hline $\bar{E} \geqslant 1$ & 7145 & 3 ron & 700 \\
\hline E 22 & 7145 & 3600 & 500 \\
\hline$E \geqslant 3$ & $884 \mathrm{H}$ & 26500 & 3500 \\
\hline E 74 & 5960 & 3000 & 1400 \\
\hline E 25 & 5880 & 4000 & 1600 \\
\hline E 26 & 5806 & 1000 & 600 \\
\hline E 27 & 5820 & 3800 & 1700 \\
\hline$E$ >0 & 5796 & 1507 & 800 \\
\hline E 29 & 5880 & 2300 & 100 \\
\hline E 30 & 7879 & 8700 & 1700 \\
\hline E 31 & 6040 & 27.00 & 700 \\
\hline 32 & 5860 & 1500 & 000 \\
\hline 33 & 7810 & 6000 & 2500 \\
\hline E 34 & 5775 & 1900 & 1100 \\
\hline$E$ 30 & 6280 & 2400 & 500 \\
\hline E 36 & $\begin{array}{l}8501 \\
8501\end{array}$ & 11100 & 2300 \\
\hline E 37 & $\begin{array}{l}\text { a } \\
\text { 61:9 }\end{array}$ & 3500 & 1800 \\
\hline$E \leqslant 0$ & 0189 & 3000 & 2300 \\
\hline E 39 & 8383 & 21900 & 3400 \\
\hline $\bar{E} 40$ & $64 h 6$ & 3 noon & 2100 \\
\hline$E 41$ & 6839 & 9799 & 1000 \\
\hline E 42 & 6205 & $1>00$ & 300 \\
\hline E 43 & 6238 & 2000 & 1500 \\
\hline E 44 & 6430 & obor. & 3000 \\
\hline E 45 & 6856 & 6001 & Jano \\
\hline E 40 & 3850 & 3100 & 2000 \\
\hline $\begin{array}{l}2 \\
E\end{array}$ & 6040 & 000 & 700 \\
\hline$E 40$ & 6856 & 3200 & 16011 \\
\hline$E 49$ & 6856 & 2000 & $8 \cap 0$ \\
\hline E bo & 6856 & 7100 & 2200 \\
\hline E 51 & 6571 & 8000 & 2500 \\
\hline E 52 & BOno & 2500 & 1700 \\
\hline E 53 & 6071 & 2200 & 900 \\
\hline E 54 & 6011 & 1700 & 110 \\
\hline E 55 & 6040 & 2000 & 500 \\
\hline 56 & 5040 & 2100 & 1000 \\
\hline E 57 & 6440 & 1400 & 700 \\
\hline$E$ So & 6440 & 2000 & 700 \\
\hline E 54 & 6865 & $3>00$ & 1900 \\
\hline bo & no 06 & 2400 & 1000 \\
\hline 61 & 6346 & $200 n$ & 1300 \\
\hline
\end{tabular}

\begin{tabular}{|c|c|c|c|}
\hline 0.58 & 0.35 & 181 & 60 \\
\hline 0.48 & 0.38 & 2.22 & 79 \\
\hline $\begin{array}{l}0.64 \\
0.93\end{array}$ & $\begin{array}{l}0.48 \\
0.60\end{array}$ & $\begin{array}{l}1.92 \\
3.54\end{array}$ & $\begin{array}{l}75 \\
64\end{array}$ \\
\hline 0.35 & 0.20 & 1.36 & 57 \\
\hline $\begin{array}{l}0.25 \\
0.25\end{array}$ & 0.08 & $? .09$ & 32 \\
\hline $\begin{array}{l}0.25 \\
0.18\end{array}$ & $\begin{array}{l}0.18 \\
0.13\end{array}$ & $\begin{array}{l}1.80 \\
1.42\end{array}$ & 78 \\
\hline 0.25 & 0.18 & 2.00 & 78 \\
\hline 1.18 & 0.48 & 5.71 & 4 \\
\hline $\begin{array}{l}0.48 \\
0.45\end{array}$ & $\begin{array}{l}0.1 H \\
0.13\end{array}$ & $\begin{array}{l}2.50 \\
2.71\end{array}$ & 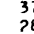 \\
\hline 0.53 & 0.25 & 1.85 & 4 \\
\hline $\begin{array}{l}.43 \\
1.20\end{array}$ & 0.05 & 2.33 & 1 \\
\hline $\begin{array}{l}1.20 \\
0.25\end{array}$ & $\begin{array}{l}0.53 \\
0.18\end{array}$ & $\begin{array}{l}\begin{array}{l}4.80 \\
3.00\end{array} \\
\text { S.00 }\end{array}$ & 74 \\
\hline 6.93 & 2.65 & 6.80 & 38 \\
\hline $\begin{array}{l}0.70 \\
0.01\end{array}$ & $\begin{array}{l}0.55 \\
1.3 \mathrm{H}\end{array}$ & $\begin{array}{l}\begin{array}{l}3.00 \\
5.81\end{array}\end{array}$ & 2 \\
\hline 0.48 & 0.20 & 2.33 & 4 \\
\hline $\begin{array}{l}1.05 \\
0.75\end{array}$ & 0.48 & 5.42 & 45 \\
\hline 32.00 & 7.40 & 7.57 & 2 \\
\hline 0.63 & 0.35 & 2.14 & $\begin{array}{l}5 \\
4 \\
4\end{array}$ \\
\hline 0.40 & 0.25 & $\because . n 6$ & 6 \\
\hline $\begin{array}{l}1.18 \\
0.30\end{array}$ & $\begin{array}{l}0.38 \\
0.13\end{array}$ & $\begin{array}{l}2.23 \\
1.87\end{array}$ & 4 \\
\hline $0.5 \mathrm{~A}$ & $\begin{array}{l}0.113 \\
0.40\end{array}$ & 3. & be \\
\hline $\begin{array}{l}\begin{array}{l}4.83 \\
0.43\end{array} \\
0.43\end{array}$ & $\begin{array}{l}2.95 \\
0.33\end{array}$ & $\begin{array}{l}5.11 \\
3.14\end{array}$ & 7 \\
\hline $0.2 \mathrm{~A}$ & 0.13 & 2.50 & 4 \\
\hline $\begin{array}{l}3.44 \\
0.16\end{array}$ & $\begin{array}{l}1.43 \\
0.03\end{array}$ & & \\
\hline 0.58 & $0.1 j$ & 4.80 & \\
\hline 7.93 & 3.01 & $4 .{ }^{\circ} 2$ & \\
\hline $\begin{array}{l}1.48 \\
2.20\end{array}$ & $\begin{array}{l}0.43 \\
1.30\end{array}$ & $\begin{array}{l}1.94 \\
1.30\end{array}$ & ? \\
\hline 16.21 & 5.75 & $n .44$ & 3 \\
\hline 1.40 & 0.50 & 1.71 & \\
\hline $\begin{array}{l}3.38 \\
0.70\end{array}$ & $\begin{array}{l}1.6 n \\
0.10\end{array}$ & $\begin{array}{r}.12 \\
4.00\end{array}$ & 5 \\
\hline 0.48 & 0.14 & 1.35 & \\
\hline 2.93 & 0.13 & 7.20 & 4 \\
\hline $\begin{array}{l}1.10 \\
0.5 B\end{array}$ & $\begin{array}{l}0.2^{n} \\
\end{array}$ & $\begin{array}{l}3.33 \\
1.55\end{array}$ & \\
\hline 0.11 & 0.07 & 0.45 & the \\
\hline $\begin{array}{l}1.95 \\
11.95\end{array}$ & $\begin{array}{l}0.33 \\
0.63\end{array}$ & $\begin{array}{l}2.00 \\
3.25\end{array}$ & \\
\hline 3.63 & 0.83 & $3 . ? 2$ & 26 \\
\hline $\begin{array}{l}3.93 \\
0.35\end{array}$ & 0.85 & $\begin{array}{l}3.20 \\
1.20\end{array}$ & $?$ \\
\hline 0.43 & $\begin{array}{l}11 \\
6.35\end{array}$ & $\begin{array}{l}1.41 \\
2.44\end{array}$ & \\
\hline 0.33 & $0.1^{5}$ & & \\
\hline 4.43 & 0.0 & & \\
\hline$\because .3 n$ & 3.05 & $?$ no & \\
\hline $0 . ?$ & $3.0 \mathrm{r}$ & & \\
\hline & 0.10 & & \\
\hline 0.31 & 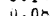 & & \\
\hline
\end{tabular}

Fig. 3. Inventory of the glaciers in the Khumbu region, Imja Khola.

carefully on maps of the drainage basin of the individual glacier shown in the map of Khumbu Himal (1: 50,000; Schneider, 1965).

The numbering system of the glaciers in the area studied is the same as Unesco/Iash (1970) by the use of the index map with glacier number by Müller (1970) which is shown in Fig. 1.

The 'inactive' ice aprons, frequent above the bergschrund, are included in the glacierized area in the guide for the glacier inventory of Unesco/ Iash (1970), however, such inactive ice aprons as fluted walls are considered to be separate from the glacier in this paper. The upper limit of a glacier (G1) was considered to be the highest position of the boundary between the active glacier and inactive ice aprons or snow walls, using aerial or terrestrial photographs and maps (Schneider, 1957 and 1965).

Length of glacier (G6) was measured on the map from the terminus to the upper limits along the center lines of the glacier.

G7 to G10 were calculated by computer using compiled data $\mathrm{G} 1 \sim \mathrm{G} 6$.
Since the smaller areas are more frequent, square root of glacier area $(\mathrm{G} 10)$ is used as an index of glacier size as well as the procedure by Nogami (1968).

On the other hand, the elements of the glacier basin are measured on the map of the area above $5500 \mathrm{~m}$, which corresponds nearly to the lower limit of precipitation in the form of snow during the warmest season, and is often about 1 $\sim 200 \mathrm{~m}$ below the firn line and the boundary between the steep wall and gentle slope. Therefore, the area of the basin above $5500 \mathrm{~m}$ seems to be suitable when considering a glacier basin from a view point of accumulation in this study.

The length of the $5500 \mathrm{~m}$ contour line (T3) is assumed to approximate the width of the basin at that altitude. The rock face area (T5) is considered in order to examine its effect on the covering of the glacier by debris, and the basin shape index $(\mathrm{T} 6 ;=\mathrm{T} 2 / \mathrm{T} 3)$ is chosen as an index of the effect of the basin shape on the gathering and concentrating of snow to feed the glacier.

The computer print-out sheets of inventory 


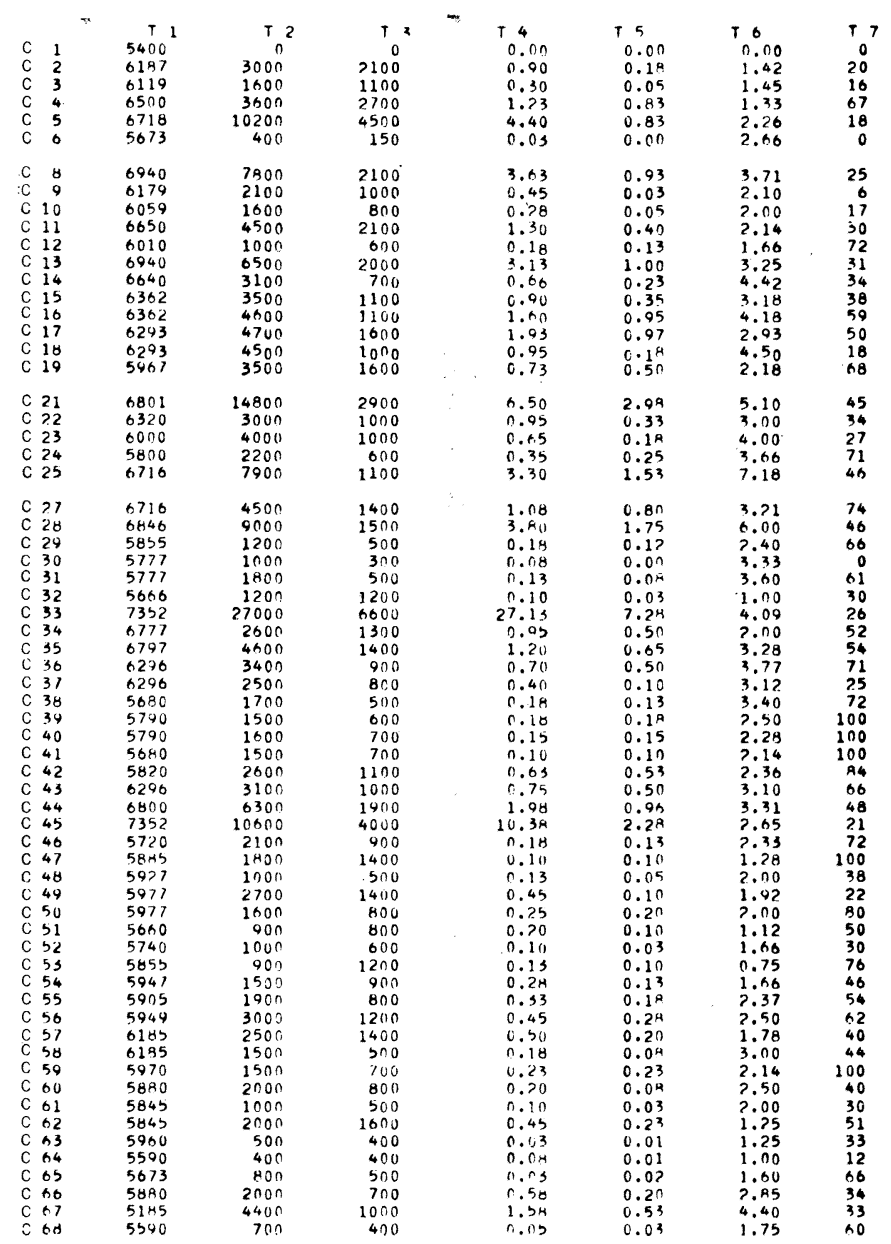

\begin{tabular}{|c|c|c|c|c|c|c|c|}
\hline 1 & 5726 & boc: & 900 & 11.115 & $0.0^{2}$ & $0 . n 6$ & 60 \\
\hline D 2 & AIRD & 210 n & ano & $n .75$ & $0.1 \mathrm{k}$ & 2.33 & 64 \\
\hline D 3 & Bi⿻s & 1600 & 300 & 2.30 & 0.23 & 5.23 & 76 \\
\hline 4 & 6073 & 5800 & $18 n 0$ & 1.012 & $0.7^{\mu}$ & 3.22 & 48 \\
\hline 5 & 0073 & 1800 & $7 n o$ & 0.23 & 0.15 & 2.57 & 65 \\
\hline 0 & 5917 & 2000 & 1200 & 0.45 & 0.30 & 1.60 & Ho \\
\hline 7 & 5977 & 2100 & 2000 & ri.34 & $0.3 n$ & 1.05 & 76 \\
\hline 8 & 5670 & 400 & 200 & $0.0 ?$ & 0.02 & $? .00$ & 100 \\
\hline D 9 & 5941 & 1900 & 1500 & 6.9B & 0.13 & 1.20 & 34 \\
\hline D 10 & 5977 & 4000 & 1600 & $n \cdot F_{0}$ & 0.33 & 7.50 & 38 \\
\hline D 11 & $\begin{array}{l}5815 \\
5815\end{array}$ & 2800 & 1200 & 0.55 & 0.20 & 2.33 & 36 \\
\hline$D 12$ & 5813 & $210 n$ & 1000 & 0.40 & 0.1 in & $? .10$ & 23 \\
\hline $\begin{array}{lll}0 & 13\end{array}$ & 5813 & $100 \%$ & 900 & 0.13 & 0.1 ? & 1.11 & 92 \\
\hline D 14 & $59 ? 2$ & ison & 700 & 6.23 & $0.0^{8}$ & 0.14 & 34 \\
\hline D 15 & 58 \% & 1900 & 000 & 0.30 & 0.20 & 3.16 & 96 \\
\hline D 16 & $5 \& 45$ & 3400 & 1500 & 1.03 & 0.43 & 2.53 & 68 \\
\hline 017 & 5600 & bo: & 300 & 0.015 & 0.011 & 2.00 & 0 \\
\hline D 19 & 6504 & 3400 & 1400 & 1.38 & $0.4 \mathrm{C}$ & 2.42 & 28 \\
\hline D 20 & 8153 & 279000 & 7900 & 50.75 & 11.44 & 3.53 & $? 2$ \\
\hline D 21 & 6010 & 2900 & 1200 & o.b: & $0.2^{11}$ & 2.41 & 33 \\
\hline D 22 & 5913 & 3100 & $15+10$ & n.tu & n.10 & 2.06 & 16 \\
\hline 023 & 5913 & $2 \mathrm{HCO}$ & 300 & 0.31 & 0.15 & 9.33 & 50 \\
\hline D 24 & 5951 & $260 ?$ & 300 & $\because .53$ & 0.20 & S.no & 36 \\
\hline D 20 & $545 \%$ & 1500 & 700 & 0.23 & 0.17 & 2.14 & 78 \\
\hline D 27 & 7020 & 19500 & 3800 & 11.40 & 4.25 & 5.13 & 35 \\
\hline$D 28$ & $58 \cap 5$ & 3700 & $1 / 00$ & $0.5 \mathrm{H}$ & $0.2^{2}$ & 2.17 & 39 \\
\hline$D>9$ & 6013 & 2800 & $7 n o$ & 0.55 & $0.2^{n}$ & 4.00 & 36 \\
\hline & DOHS & 2600 & 1400 & 0.53 & $0.2^{4}$ & 1.85 & 52 \\
\hline 31 & OOR9 & 1400 & 900 & 0.25 & 0.22 & 1.55 & 88 \\
\hline 32 & 5931 & $110 n$ & 4010 & 0.35 & 0.29 & 1.22 & 84 \\
\hline 33 & 6440 & 3400 & 1300 & 0.85 & 0.13 & 2.92 & 15 \\
\hline 34 & 5700 & 700 & 600 & $n .05$ & 0.03 & 1.16 & nO \\
\hline D 35 & 6361 & $550 \mathrm{n}$ & 1700 & 1.18 & 0.33 & 3.23 & 27 \\
\hline C 36 & $654 ?$ & 4500 & 17001 & 1.00 & 0.60 & 2.04 & 60 \\
\hline D 37 & 50110 & 600 & Si) 0 & 0.02 & 0.02 & 1.20 & 100 \\
\hline
\end{tabular}

Fig. 4. Inventory of the glacier basins in the Khumbu region, Bhote Kosi and Dudh Kosi. 


\begin{tabular}{|c|c|c|c|}
\hline 1 & 5500 & 5280 & 5280 \\
\hline 2 & 5400 & 5100 & 5100 \\
\hline $\begin{array}{ll}E & 3 \\
F & 4\end{array}$ & $\begin{array}{l}5200 \\
5600\end{array}$ & $\begin{array}{l}4580 \\
5020\end{array}$ & $\begin{array}{l}4860 \\
5020\end{array}$ \\
\hline $\begin{array}{ll}E & 4 \\
E & 5\end{array}$ & $\begin{array}{l}5600 \\
5400\end{array}$ & $\begin{array}{l}5020 \\
5040\end{array}$ & $\begin{array}{l}5020 \\
5040\end{array}$ \\
\hline 0 & 5360 & 4740 & 4840 \\
\hline 7 & 5620 & 5100 & 5100 \\
\hline 8 & $\begin{array}{l}5500 \\
5500\end{array}$ & 5160 & 5160 \\
\hline$E_{10}$ & $\begin{array}{l}5560 \\
5670\end{array}$ & 5280 & 5280 \\
\hline$E{ }_{11}$ & 5600 & $\begin{array}{l}5180 \\
5300\end{array}$ & $\begin{array}{l}5120 \\
5300\end{array}$ \\
\hline 12 & 5600 & 5160 & $\begin{array}{l}5300 \\
5100\end{array}$ \\
\hline 13 & 5400 & 5140 & $5: 40$ \\
\hline 14 & 5840 & 5140 & 5140 \\
\hline$E$ is & 5840 & 4980 & 5119 \\
\hline E 10 & 5840 & 5540 & 5540 \\
\hline$E 17$ & 6040 & 5220 & 5280 \\
\hline $\begin{array}{ll}E & 18\end{array}$ & 5680 & 5300 & 5440 \\
\hline 19 & n200 & 5119 & 5290 \\
\hline 20 & 5840 & 5440 & 5440 \\
\hline$? 1$ & 6000 & 5320 & 5440 \\
\hline 22 & tono & 5520 & 5520 \\
\hline 23 & 7400 & 4920 & 5220 \\
\hline 24 & 5900 & 52710 & 5270 \\
\hline$E 2.5$ & 5800 & sion & $5100^{\circ}$ \\
\hline 26 & 5800 & 5360 & 5360 \\
\hline 27 & 5770 & $554 n$ & 5540 \\
\hline 28 & 5640 & 5380 & $53 H 0$ \\
\hline 29 & 5440 & 3580 & 5580 \\
\hline 30 & 5800 & 4970 & 5480 \\
\hline 31 & 5650 & 5420 & 5470 \\
\hline 32 & 5750 & 5380 & $53 \times 0$ \\
\hline 33 & 5800 & 4980 & 51100 \\
\hline 34 & $54 R 0$ & 5250 & 5250 \\
\hline 55 & bono & 5400 & 5400 \\
\hline 30 & 58 ino & 4920 & 5200 \\
\hline 37 & 0179 & 5759 & 5259 \\
\hline E 38 & 6179 & 5360 & 5560 \\
\hline 30 & AONO & 5020 & 5280 \\
\hline 40 & 6300 & 5250 & 5259 \\
\hline 41 & 5600 & sion & 5254 \\
\hline 42 & 5700 & $53 \mathrm{HO}$ & $53 \mathrm{KO}$ \\
\hline$E 43$ & 5400 & 5020 & 5020 \\
\hline E 44 & 6050 & $502 \%$ & 5020 \\
\hline$E 4 b$ & 5000 & $4 B 20$ & 5100 \\
\hline$E 40$ & 5400 & 4780 & 4920 \\
\hline$E 47$ & 5300 & 4520 & 4740 \\
\hline 48 & 5800 & 5100 & 5100 \\
\hline E 49 & 5800 & 5300 & 5460 \\
\hline 50 & 5880 & 5020 & 5340 \\
\hline 51 & 5919 & $5 \cap 2 n$ & 5140 \\
\hline 52 & 5000 & $4 \pi 2 n$ & 4900 \\
\hline 53 & 5500 & 4580 & 5000 \\
\hline 54 & 5254 & 4780 & 4820 \\
\hline 55 & 5259 & $474 n$ & 4780 \\
\hline $\begin{array}{l}56 \\
57\end{array}$ & 5300 & 4620 & 5000 \\
\hline 57 & 5400 & 4840 & 4840 \\
\hline 58 & $54 \pi 0$ & 4860 & 4860 \\
\hline 59 & 5300 & 4?? & $45 ? 0$ \\
\hline 60 & 5900 & 5320 & 5320 \\
\hline A1 & 5240 & 4420 & $48>0$ \\
\hline
\end{tabular}

\begin{tabular}{|c|c|c|}
\hline $\begin{array}{l}0.00 \\
0.00\end{array}$ & $\begin{array}{l}0.05 \\
0.33\end{array}$ & 400 \\
\hline $\begin{array}{l}0.00 \\
0.89\end{array}$ & $\begin{array}{l}0.33 \\
1.27\end{array}$ & 2900 \\
\hline 0.00 & 0.29 & GOO \\
\hline 0.00 & 0.05 & 500 \\
\hline 0.20 & $0.3 ?$ & 1200 \\
\hline 0.06 & 0.17 & HOO \\
\hline 0.00 & 0.10 & 500 \\
\hline 0.00 & 0.41 & 1400 \\
\hline 0.00 & 0.95 & 2000 \\
\hline 0.00 & 0.19 & 700 \\
\hline 0.00 & 0.20 & nOO \\
\hline 0.00 & 0.27 & 500 \\
\hline 0.00 & 0.39 & 1000 \\
\hline 0.70 & 1.40 & 2100 \\
\hline 0.00 & 0.07 & 400 \\
\hline$? .98$ & 8.28 & 7000 \\
\hline 0.51 & 0.92 & $2>00$ \\
\hline 2.53 & 5.96 & 4500 \\
\hline 11.00 & 0.27 & AOO \\
\hline 0.85 & 1.24 & 2000 \\
\hline 0.00 & 0.25 & noo \\
\hline 8.27 & 19.01 & 16500 \\
\hline 0.00 & 0.42 & 900 \\
\hline 0.00 & 0.69 & 2100 \\
\hline 0.00 & 0.13 & 400 \\
\hline $0 . n 0$ & 0.17 & 800 \\
\hline 0.00 & 0.13 & 500 \\
\hline$n \cdot n o$ & 0.11 & 400 \\
\hline 3.50 & $4.8 ?$ & OMOO \\
\hline 0.00 & 0.20 & 500 \\
\hline 1.00 & 0.13 & noo \\
\hline 2.37 & 3.32 & 4500 \\
\hline 0.07 & 0.17 & 500 \\
\hline 0.011 & 0.15 & 700 \\
\hline 5.55 & $7.2 ?$ & 6300 \\
\hline 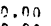 & 0.95 & 1000 \\
\hline 0.00 & 0.24 & 700 \\
\hline 7.09 & 10.99 & 11200 \\
\hline 0.00 & 0.80 & 1000 \\
\hline 1.01 & 3.04 & 3500 \\
\hline 0.00 & 0.04 & 600 \\
\hline 0. no & 0.09 & $50 n$ \\
\hline 0.00 & 4.73 & 2300 \\
\hline 2.58 & 3.20 & 4500 \\
\hline 1.19 & 1.51 & $2 \mathrm{H} 00$ \\
\hline 0.47 & 0.73 & $2 ? 00$ \\
\hline n.no & 0.74 & 1400 \\
\hline 0.313 & 0.41 & 1600 \\
\hline 7.34 & 4.34 & $3 \mathrm{HOO}$ \\
\hline$n .35$ & 3.67 & 2200 \\
\hline 1.11 & 1.63 & non \\
\hline 0.20 & 0.34 & ROO \\
\hline 0.04 & 0.15 & 500 \\
\hline$n .03$ & $0.1^{2}$ & 900 \\
\hline $0.2 \mathrm{~A}$ & 0.47 & 500 \\
\hline n.no & 0.16 & 900 \\
\hline 0.00 & 0.1 A & 11100 \\
\hline 0.18 & 1.17 & lagr \\
\hline 0.00 & 0.31 & 400 \\
\hline$n .00$ & 0.20 & $90 n$ \\
\hline
\end{tabular}

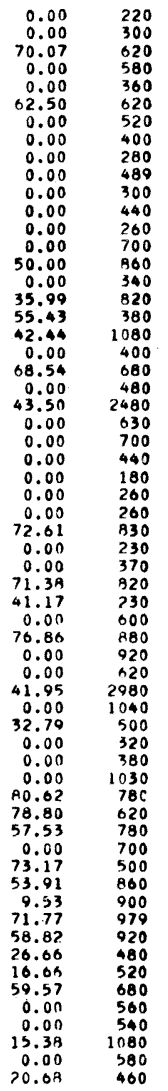

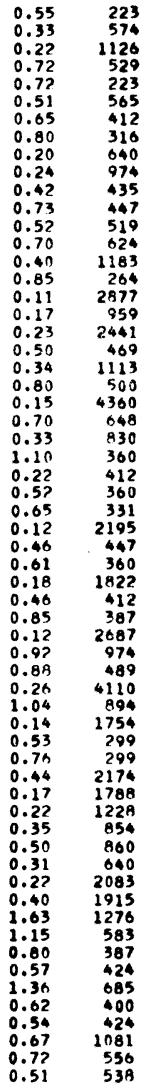

Fig. 5. Inventory of the glacier basins in the Khumbu region, Imja Khola.

data of the glaciers and their basins in the Khumbu region are shown in Figs. $2 \sim 5$.

\section{Statistical analysis of glacier forms and their relation to basin morphology.}

1) Preliminary statistics of glacier inventory.

Two classifications of the glaciers in the Nepal Himalayas were proposed by Tanaka (1971) and Moribayashi (1973) based on their field observations in Khumbu Himal. Tanaka classified them into the Lower Himalayan type developed below $6000 \mathrm{~m}$ and the Higher Himalayan type formed in the altitudinal range $5000 \sim 7000 \mathrm{~m}$. Moribayashi proposed to call the debris-covered glaciers "Dirty type (D-type) glaciers" and the debris-free glaciers "Clean type (C-tyep) glaciers".

Table 1. shows the results of a statistical analysis of the glacier inventory in the Khumbu region. As seen in this table, C-type glaciers develop in the altitudinal range between $5250 \mathrm{~m}$ and $5700 \mathrm{~m}$ and have an area of $0.4 \mathrm{~km}^{2}$ on the average. On the other hand, D-type glaciers develop from $4890 \mathrm{~m}$ to $5960 \mathrm{~m}$, more than double the altitudinal range of C-type glaciers, and have an area of $6.6 \mathrm{~km}^{2}$ on the average, more than fifteen times the C-type glaciers area. The mean length of D-type glaciers, $5.2 \mathrm{~km}$, is more than five times as long as that of C-type glaciers, 1.0 $\mathrm{km}$.

It is, therefore, clear that there is an apparent difference in the dimensions of C- and D-type glaciers in the Khumbu Himal.

Each element of basins containing D-type glaciers, especially length of basin ridge and basin area, is bigger than the corresponding element for C-type glaciers, and so is like to be more effective for glacierization.

The total area of glaciers and the total number of glaciers in the Khumbu region are $287 \mathrm{~km}^{2}$ and 167 respectively.

2) Correlation coefficients between the dimensions of glaciers and their basins.

Correlation coefficients between the dimensions of glaciers and their basins were computed and the results are shown in Tables 2 and 3.

In general, the correlation is better in the case 
Table 1. Elements representative of the forms of glaciers and glacier basins in the Khumbu region.

\begin{tabular}{|c|c|c|c|c|c|}
\hline \multirow{2}{*}{\multicolumn{2}{|c|}{$\begin{array}{l}\text { Elements of glacier and its } \\
\text { basin above } 5500 \mathrm{~m}\end{array}$}} & \multicolumn{2}{|c|}{$\begin{array}{l}\text { C-type glacier } \\
\text { with G5 above } 0.1 \mathrm{~km}^{2} \text { (89) }\end{array}$} & \multicolumn{2}{|c|}{$\begin{array}{l}\text { D-type glacier } \\
\text { with G4 above } 0.1 \mathrm{~km}^{2} \text { (37) }\end{array}$} \\
\hline & & MEAN & ST. DEVIATION & MEAN & ST. DEVIATION \\
\hline U. LIMIT (m) & G1 & 5706 & 237 & 5963 & 639 \\
\hline L. LIMIT (m) & G2 & 5247 & 187 & 4886 & 289 \\
\hline EXP. LIMIT (m) & G3 & & & 5114 & 255 \\
\hline DEBRIS AREA $\left(\mathrm{km}^{2}\right)$ & $\mathrm{G}_{4}$ & & & 2. 72 & 4. 10 \\
\hline TOTAL AREA $\left(\mathrm{km}^{2}\right)$ & G5 & 0.43 & 0.55 & 6.55 & 11.50 \\
\hline LENGTH $(\mathrm{km})$ & G6 & 0.96 & 0.49 & 5117 & 4. 99 \\
\hline MAX. HEIGHT (m) & $\mathrm{T} 1$ & 6090 & 325 & 6937 & 700 \\
\hline RIDGE LENGTH (m) & $\mathrm{T} 2$ & 2555 & 1232 & 8816 & 7223 \\
\hline VALLEY WIDTH (m) & T3 & 1080 & 529 & 2222 & 1544 \\
\hline BASIN AREA $\left(\mathrm{km}^{\natural}\right)$ & $\mathrm{T} 4$ & 0.62 & 0.52 & 6.17 & 10.31 \\
\hline ROCK F. AREA $\left(\mathrm{km}^{2}\right)$ & $\mathrm{T} 5$ & 0.26 & 0.24 & 1.88 & 2.47 \\
\hline BASIN S. INDEX & T6 & 2. 60 & 1. 43 & 3.85 & 1. 71 \\
\hline ROCK F.A. RATIO (\%) & $\mathrm{T} 7$ & 45 & 24 & 43 & 18 \\
\hline
\end{tabular}

Table 2. Correlation Coefficients between the dimensions of the debris-free glaciers and their basins.

\begin{tabular}{lc|c|r|r|r|r|r|r}
\hline & & $\begin{array}{l}\text { MAX. } \\
\text { HEIGHT } \\
\text { T1 }\end{array}$ & $\begin{array}{l}\text { RIDGE } \\
\text { LENGTH } \\
\text { T2 }\end{array}$ & $\begin{array}{l}\text { VALLEY } \\
\text { WIDTH } \\
\text { T3 }\end{array}$ & $\begin{array}{c}\text { BASIN } \\
\text { AREA } \\
\text { T4 }\end{array}$ & $\begin{array}{l}\text { ROCK } \\
\text { F. AREA } \\
\text { T5 }\end{array}$ & $\begin{array}{l}\text { BASIN } \\
\text { S.INDEX } \\
\text { T6 }\end{array}$ & $\begin{array}{l}\text { ROCK } \\
\text { F.A.RATIO } \\
\text { T7 }\end{array}$ \\
\hline U.LIMIT & G1 & & 0.40 & 0.30 & 0.50 & 0.31 & 0.16 & -0.29 \\
L.LIMIT & G2 & & 0.06 & 0.08 & 0.06 & 0.25 & 0.27 & 0.23 \\
T.AREA & G5 & 0.28 & 0.65 & 0.56 & 0.71 & 0.17 & 0.03 & -0.30 \\
LENGTH & G6 & 0.23 & 0.64 & 0.33 & 0.49 & 0.22 & 0.15 & -0.25 \\
AL.RANGE & G8 & 0.52 & 0.39 & 0.40 & 0.49 & 0.12 & -0.06 & -0.51 \\
GRADIENT & G9 & 0.24 & -0.17 & 0.14 & 0.03 & -0.01 & -0.23 & -0.20 \\
ROOT T.AREA & G10 & 0.37 & 0.74 & 0.60 & 0.73 & 0.28 & 0.04 & -0.36 \\
\hline
\end{tabular}

(on 89 glaciers with an area above $0.1 \mathrm{~km}^{2}$ )

Table 3. Correlation Coefficients between the dimensions of the debris-covered glaciers and their basins.

\begin{tabular}{ll|r|r|r|r|r|r|r}
\hline & & $\begin{array}{l}\text { MAX. } \\
\text { HEIGHT } \\
\text { T1 }\end{array}$ & $\begin{array}{l}\text { RIDGE } \\
\text { LENGTH } \\
\text { T2 }\end{array}$ & $\begin{array}{l}\text { VALLEY } \\
\text { WIDTH } \\
\text { T3 }\end{array}$ & $\begin{array}{l}\text { BASIN } \\
\text { AREA } \\
\text { T4 }\end{array}$ & $\begin{array}{l}\text { ROCK } \\
\text { F. AREA } \\
\text { T5 }\end{array}$ & $\begin{array}{l}\text { BASIN } \\
\text { S.INDEX } \\
\text { T6 }\end{array}$ & $\begin{array}{l}\text { ROCK } \\
\text { F. RATIO } \\
\text { T7 }\end{array}$ \\
\hline U.LIMIT & G1 & & 0.82 & 0.66 & 0.75 & 0.77 & 0.51 & -0.45 \\
L.LIMIT & G2 & & -0.02 & -0.29 & -0.10 & -0.04 & 0.51 & 0.15 \\
EXP.LIMIT & G3 & & 0.15 & -0.12 & 0.06 & 0.15 & 0.53 & 0.19 \\
DEBRIS AREA & G4 & 0.65 & 0.85 & 0.87 & 0.95 & 0.94 & 0.25 & -0.39 \\
TOTAL AREA & G5 & 0.56 & 0.82 & 0.89 & 0.96 & 0.93 & 0.21 & -0.40 \\
LENGTH & G6 & 0.70 & 0.94 & 0.85 & 0.93 & 0.95 & 0.44 & -0.43 \\
DEBRIS A.RATO & G7 & 0.01 & -0.31 & -0.31 & -0.21 & -0.19 & -0.21 & 0.36 \\
AL.RANGE & G8 & 0.06 & 0.81 & 0.78 & 0.78 & 0.77 & 0.28 & -0.51 \\
GRADIENT & G9 & -0.48 & -0.41 & -0.29 & -0.29 & -0.35 & -0.46 & 0.26 \\
ROOT T.AREA & G10 & 0.67 & 0.93 & 0.94 & 0.94 & 0.95 & 0.38 & -0.53 \\
\hline
\end{tabular}

(on 37 glaciers with a debris covered area above $0.1 \mathrm{~km}^{2}$ ) 
Table 4. Multiple regression equation of the debris-covered and debris-free glaciers, using the elements representing the forms of the glacier basins.

a) C-type glacier with an area above $0.1 \mathrm{~km}^{2}$ (89 glaciers)

G1 $(\mathrm{m})=5135+190 \times \mathrm{T} 4-1.04 \times \mathrm{T} 7+0.08 \times \mathrm{T} 1$

$\mathrm{G} 2 \quad(\mathrm{~m})=5018+39.70 \times \mathrm{T} 6+1.98 \times \mathrm{T} 7+131 \times \mathrm{T} 5$

G5 $\left(\mathrm{km}^{2}\right)==-0.25+1.56 \times \mathrm{T} 4-2.19 \times \mathrm{T} 5+0.0062 \times \mathrm{T} 7$

0.52

$\mathrm{G} 6 \quad(\mathrm{~m})=2.25+0.0003 \times \mathrm{T} 2-0.50 \times \mathrm{T} 5-0.0003 \times \mathrm{T} 1$

b) D-type glacier with a debris covered area above $0.1 \mathrm{~km}^{2}$ (37 glaciers)

$\mathrm{G} 1(\mathrm{~m})=4249+0.06 \times \mathrm{T} 2+0.16 \times \mathrm{T} 1+21.68 \times \mathrm{T} 6$

$\mathrm{G} 2 \quad(\mathrm{~m})=4574+134.71 \times \mathrm{T} 6-0.03 \times \mathrm{T} 2+45.38 \times \mathrm{T} 5$

G3 $(\mathrm{m})=4237+81.81 \times \mathrm{T} 6+5.31 \times \mathrm{T} 7+0.05 \times \mathrm{T} 1$

G4 $\left(\mathrm{km}^{2}\right)=-0.72+0.15 \times \mathrm{T} 4+0.0005 \times \mathrm{T} 3+0.63 \times \mathrm{T} 5$

G5 $\left(\mathrm{km}^{2}\right)=-1.27+1.05 \times \mathrm{T} 4+0.0024 \times \mathrm{T} 3-0.0004 \times \mathrm{T} 2$

G6 $(\mathrm{m})=0.29+1.14 \times \mathrm{T} 5+0.0002 \times \mathrm{T} 2+0.0003 \times \mathrm{T} 3$

0.96

0.98

0.97

of D-type glaciers than in that of C-type glaciers. The reason will be discussed in the next section.

The large-sized glaciers, having a large area, long length, and high upper limit, develop in bigger basins with higher summits, longer ridges and wider valleys.

The correlations between glacier area and basin area; glacier length and ridge length; and debris covered area and rock face area are especially good.

Therefore, it would be reasonable to consider that the total precipitation in the basin above the firn line controls the size of the glacier, and the debris on the glacier surface was supplied from the rock face of the basin.

The position of the lower limit of the glacier is independent the dimensions of the glacier basin, as in the case of the Cordillera Real, Bolivia (Nogami, 1968).

3) Multiple regression analysis of glacier forms

A multiple regression analysis of glacier forms was performed as a pilot study to relate glacier form to some basin factors by a regression equation.

The program of this analysis is for a single dependent and a maximum of 49 explaining variables. The technique used is the so-called forward selection method. Explaining variables are added to the regression equation one at a time. At each step, the program selects that remaining variable which makes the greatest improvement in 'goodness of fit', that is the greatest reduction in the residual sum of squares.

The results are shown in Table 4. Each regression equation is shown with three explaining variables and the multiple correlation coefficients are greater in the case of D-type glaciers than in the case of C-type glaciers. This may indicate that the forms of D-type glaciers which have dead ice in terminal part are close to the forms attained during the maximum stage in the last advance. On the other hand, the forms of C-type glaciers are different in each glacier which is in response to present or past climatic conditions, after separation from debris covered dead ice body by melting (Fushimi, 1977) depending on the individual size of the glacier, so that the coefficient is smaller than in that of D-type glaciers.

\section{References}

Fushimi, H. (1977): Structural studies on glaciers in the Khumbu Region. in this issue.

Moribayashi, S. (1973): Nepal Himalaya no Hyôga ni tsuite sono tokusei to saikin no hendô (On the Characteristics of Nepal Himalayan Glaciers and Their Recent Variation). Seppyo, 36, 1, 11-21.

Müller, F. (1970): Inventory of glaciers in the Mount Everest region. In Technical Papers in Hydrology 1-Perennial ice and snow masses, UNESCO/IASH, 47-59.

Nogami, M. (1968): Cordillera Real (Bolivia) no hyôga (Statistical Analysis of the altitudinal Distribution of Glaciation in the Cordillera Real, Bolivia). Journal of Geography, 77, 125-140.

Schneider, E. (1957): Mahalangur Himal (1: 25,000), Kartographische Anstalt Freytag-Berndt und Artaris, Wien.

Schneider, E. (1965): Khumbu Himal (1: 50,000), Kartographische Anstalt Freytag-Berndt und Artaris, Wien.

Tanaka, H. (1971): On Preferred Orientation of Glacier and Experimentally Deformed Ice. A thesis for a master's degree of Facuity of Science, Hokkaido University.

UNESCO/IASH, (1970): Technical papers in hydrology 1.- Perennial ice and snow masses, 59. 